\title{
ANÁLISIS DE LA POSTURA DEL TRONCO EN TERAPIAS DE REHABILITACIÓN MEDIANTE ROBOTS
}

\author{
Aitziber Mancisidor ${ }^{1}$ Asier Brull ${ }^{1}$ Asier Zubizarreta ${ }^{1}$ Itziar Cabanes $^{1}$ Eva Portillo ${ }^{1}$ \\ Ana Rodriguez ${ }^{2}$ Rakel Berriozabalgoitia ${ }^{2}$ \\ \{aitziber.mancisidor, abrull001, asier.zubizarreta, itziar.cabanes, eva.portillo, ana.rodriguez\}@ehu.eus \\ ${ }^{1}$ Departamento de Ingeniería de Sistemas y Automática, Escuela de Ingeniería de Bilbao (UPV/EHU) \\ ${ }^{2}$ Departamento de Fisiología, Facultad de Medicina y Enfermería (UPV/EHU)
}

\section{Resumen}

Conocer la postura del tronco de los pacientes durante las terapias de rehabilitación, permite al terapeuta realizar un análisis más exhaustivo de su estado de recuperación funcional y adecuar los ejercicios a sus necesidades. Sin embargo, la mayoría de los robots de rehabilitación de miembros superiores, no poseen la capacidad de monitorizar la postura del tronco de los usuarios. Ante esta situación, este trabajo presenta un innovador software de rehabilitación que permite al terapeuta conocer la postura del tronco del paciente, la posición de la extremidad afectada y el porcentaje de movimiento realizado con el tronco. El software propuesto se ha implementado en el robot de rehabilitación de extremidades superiores UHP. Con la intención de evaluar su uso, se han llevado a cabo diferentes pruebas experimentales con un grupo de 25 personas sanas. Los resultados muestran que el software propuesto funciona correctamente al medir el movimiento del tronco.

Palabras clave: Rehabilitación mediante dispositivos robóticos, rehabilitación de extremidades superiores, análisis de la postura del tronco, validación experimental.

\section{INTRODUCCIÓN}

El accidente cerebrovascular o el ictus es una de las enfermedades con mayor incidencia de hoy en día. Cada año se diagnostican más de 15 millones de nuevos casos [4]. Además, debido al envejecimiento global de la población, se prevée que en los próximos años se incremente el número de personas afectadas por esta enfermedad. Se estima que para el año 2030 el número de ataques anuales llegue hasta los 23 millones [10].

En los últimos años, gracias a los nuevos protocolos médicos y terapéuticos, se ha conseguido aumentar la tasa de supervivencia, lo que ha incrementado el número de pacientes afectados por un ataque de ictus [5]. Hoy en día, cerca de 33 millones de personas en el mundo tiene que vivir con alguna secuela debido a esta enfermedad [13], donde la perdida de movilidad es una de las secuelas más frecuentes. Se estima que más del $80 \%$ de los pacientes afectados por ictus sufren algún déficit en su sistema motor [12].

En ausencia de un tratamiento quirúrgico o farmacológico, la rehabilitación se ha vuelto esencial para mejorar la calidad de vida de los pacientes con movilidad reducida debido a un ictus. La rehabilitación de las funciones perdidas es un proceso largo que requiere que el terapeuta diagnostique y trate al paciente de forma individualizada y continua [7]. Lo que genera que en los programas tradicionales, el período de rehabilitación este restringido debido a la limitación temporal del personal y los recursos económicos [14]. Debido a estas limitaciones, los programas de recuperación convencionales priorizan la recuperación de las extremidades inferiores con el objetivo de conseguir la autonomía para caminar de los pacientes. No obstante, la movilidad de los miembros superiores es fundamental para llevar a cabo actividades de la vida diaria, siendo su rehabilitación fundamental para reducir los niveles de discapacidad del paciente. Es por ello por lo que en los últimos años se han propuestos diversos dispositivos robóticos para participar en la rehabilitación de las extremidades superiores $[1,11,17]$.

Por lo general, las personas con movilidad reducida usan el tronco del cuerpo para compensar la falta de movilidad de sus extremidades. Por ello, en terapias de rehabilitación de las extremidades superiores con dispositivos robóticos, es importante conocer la postura del tronco de los pacientes [15]. Por lo que la monitorización del movimiento del tronco durante las terapias puede proporcionar información sobre el estado de recuperación del paciente permitiendo al terapeuta adecuar los ejercicios a sus necesidades. Al mismo tiempo, conocer la postura del tronco previene posturas inapropiadas que pueden lesionar a los pacientes y aumentar el tiempo de recuperación [6].

Sin embargo, la mayoría de los robots de rehabilitación de miembros superiores existentes, no poseen la capacidad de medir y monitorizar la postura del tronco [2]. Los robots basados en el contacto con el elemento terminal presentan un único punto de contacto con el paciente, por lo que es casi 
imposible conocer la postura exacta del tronco así como del miembro afectado [16]. Por otro lado, los exoesqueletos permiten controlar y monitorizar la posición de la extremidad a rehabilitar, pero no posibilitan analizar el movimiento del tronco [3].

En vista de esta situación, este trabajo presenta un innovador software de rehabilitación que permite al terapeuta conocer la postura del tronco del paciente y estimar el porcentaje de movimiento realizado con el tronco. Este software ha sido implementado en el robot de rehabilitación UHP [9] y validado con 25 usuarios sanos.

El resto del artículo está estructurado de la siguiente manera. En la Sección II se describe el software para el análisis de la postura del tronco. En la Sección III, el software propuesto se implementa en el robot de rehabilitación UHP. En la sección IV se evaluá el software propuesto. Finalmente, las ideas más importantes y los trabajos futuros se resumen en las conclusiones.

\section{SOFTWARE PARA EL ANÁLISIS DE LA POSTURA DEL TRONCO}

El software propuesto para el análisis de la postura del tronco tiene dos objetivos: 1) Medir y visualizar la postura del tronco de los pacientes con movilidad reducida; 2) Estimar la contribución del cuerpo durante la terapia de rehabilitación.

\subsection{Medición y visualización de la postura del tronco}

Con el fin de medir la postura del tronco, se han agregado dos unidades de medición inerciales o IMUs (Inertial Measurement Unit). Los sensores IMU utilizados se encuentran incorporados en placas comercializadas MPU IMU Click (MPU-6000 de InvenSense), que permiten medir el ángulo de rotación en los tres ejes. Para la correcta adecuación de los sensores IMU al cuerpo del paciente se han fabricado dos cajas mediante impresión 3D. Estas cajas, en las cuales se han incorporado el sensor IMU, se unen a la espalda y a la parte posterior del brazo del usuario fácilmente a través de cintas con velcro (Figura 1).

La información de los sensores IMUS se trata y se muestra en pantalla a través de una interfaz gráfica programada con Labview-RT de National Instrument. La Figura 2 muestra la interfaz gráfica del software propuesto que consiste en un avatar 3D similar al humano y cuatro gráficos de los datos procesados del sensor, cuales proporcionan una interpretación intuitiva al terapeuta.
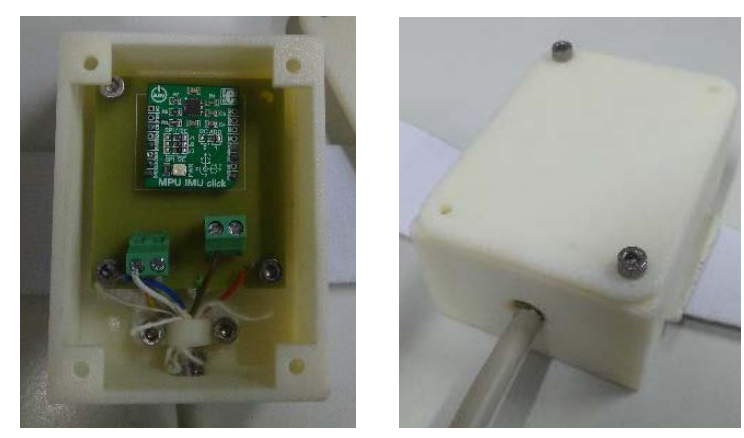

Figura 1: Caja para adecuar al cuerpo la IMU.

El avatar 3D del lado izquierdo de la Figura 2 reproduce el movimiento del tronco y de la extremidad superior del usuario. Las flechas colocadas debajo del avatar se iluminan cuando el paciente mueve el tronco más de lo deseado. Mediante esta interfaz gráfica, el paciente puede visualizar cuando está utilizando el tronco más de lo permitido y tratar de minimizar el error cometido.

Por otro lado, en el lado derecho de la Figura 2, hay cuatro gráficos que indican la orientación mediolateral (inclinación en el eje $z$ ) y fronto-trasera (inclinación en el eje $x$ ) del tronco y de la extremidad superior afectada. Con el fin de que el terapeuta pueda analizar la implicación del tronco del paciente durante toda la terapia de rehabilitación, las mediciones de inclinación, además de mostrarlas en pantalla, se registran en la memoria del ordenador.

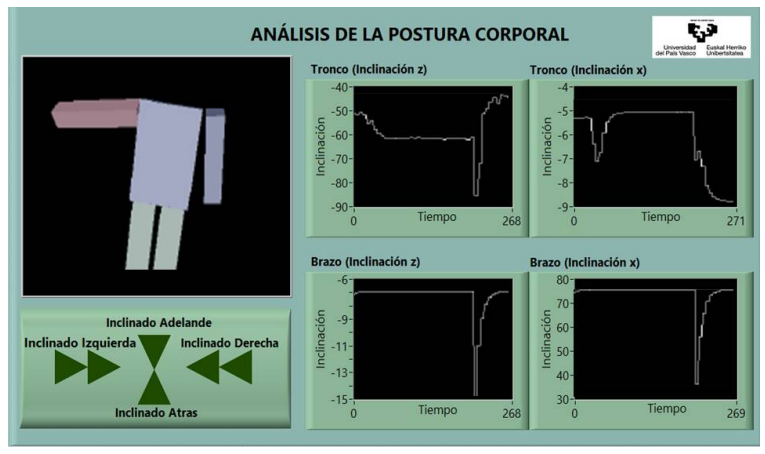

Figura 2: Interfaz gráfica para el análisis de la postura del tronco.

\subsection{Estimación de la contribución del tronco}

Otra funcionalidad implementada en el software es proporcionar el porcentaje de la participación del tronco en la ejecución de los ensayos de rehabilitación. Con este fin, a partir de las mediciones de los IMUS, conociendo la posición del robot y los rasgos físicos de los sujetos (estatura y longitud del brazo), se ha estimado la posición exacta 
del codo y del hombro. Y de esta estimación, se ha obtenido el aporte del tronco en cada movimiento. Es decir, se ha calculado el porcentaje del movimiento ejecutado con la extremidad superior y con el tronco.

\section{IMPLEMENTACIÓN EN EL ROBOT UHP}

\subsection{Robot de rehabilitación UHP}

El software propuesto se ha implementado en el robot de rehabilitación UHP (Universal Haptic Pantograph) [9]. Como se puede observar en la Figura 3, el UHP es un dispositivo robótico diseñado para la rehabilitación de las extremidades superiores. Es decir, posibilita rehabilitar las articulaciones del hombro, codo y muñeca. Entre los diferentes modos de operación que posee, en este trabajo se ha utilizado el modo ARM que permite al robot realizar movimientos planares, los cuales se traducen en ejercicios de flexión/extensión de prolongación y apertura del brazo. Esto posibilita realizar ejercicios de rehabilitación asociados a los tres movimientos del hombro (rotación, flexión/extensión y abducción/adducción) y el movimiento flexión/extensión del codo [8].

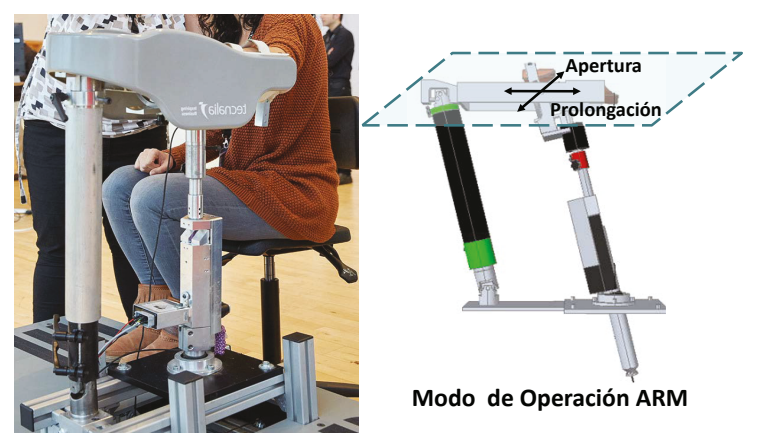

Figura 3: Robot de rehabilitación de UHP.

El robot de rehabilitación UHP, en el modo de operación ARM posee un juego de rehabilitación que divide el área de trabajo del robot en 5 regiones. Como se puede observar en la Figura 4, el eje vertical del juego corresponde al movimiento de prolongación del brazo, mientras que el movimiento de apertura se visualiza en el eje horizontal.

En el juego, el paciente debe mover el robot desde el punto de origen $\left(\mathbf{P}_{\mathbf{I n i}}\right)$ hasta el extremo de la región deseada resaltada en rojo, y volver al punto de origen. Una vez regresado al punto de origen, el juego activa otra nueva región aleatoriamente, y el paciente debe repetir el proceso hasta finalizar el tiempo de entrenamiento seleccionado por el terapeuta.

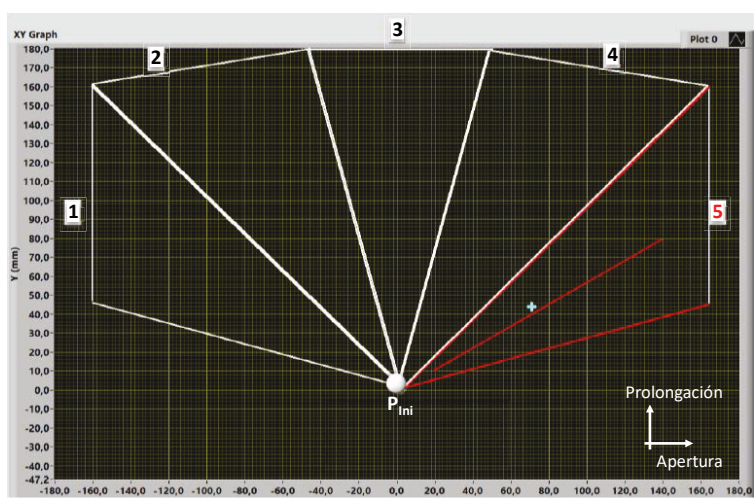

Figura 4: Juego de rehabilitación implementado en el robot UHP.

\subsection{Diseño de los ensayos de evaluación}

Con el fin de evaluar el software propuesto, se han ejecutado diferentes ensayos con 25 personas sanas y el robot de rehabilitación UHP. Para una evaluación correcta, el rango de movimiento del juego de rehabilitación ha sido adaptado a las características físicas de cada participante. Además, se ha utilizado el mismo orden de activación de regiones para todos los usuarios.

En la ejecución de estos ensayos, con la intención de generar un entorno realista, se ha tratado de simular a pacientes con movilidad reducida. Con este objetivo, se ha limitado la movilidad de las dos articulaciones que se ejercen en el modo de operación ARM, es decir, se ha restringido el movimiento del codo y del hombro. Con el fin de reducir el movimiento del codo se ha utilizado una codera que consta de una serie de sujeciones que restringen el movimiento de extensión del codo. Por otro lado, el movimiento del hombro se ha limitado mediante una cinta que ha rodeado el cuerpo del participante sujetando los dos hombros contra el tronco (Figura 5). Por lo que cada participante ha realizado tres tipos de ensayos de evaluación: sin restricciones físicas, con restricción del movimiento del codo y con restricción del movimiento del hombro.
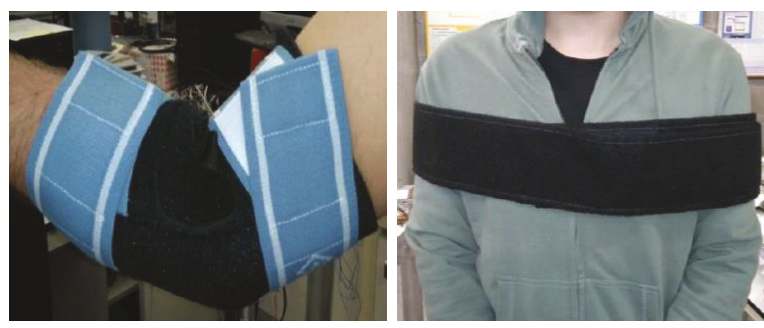

Figura 5: Restricciones físicas del movimiento del codo y del hombro. 
Por otro lado, se ha considerado que el respaldo de la silla puede repercutir en la posición del tronco del usuario durante la ejecución de los ejercicios de rehabilitación. Por ello, los ensayos definidos ( $\sin$ restricciones físicas, con restricción del movimiento del codo y con restricción del movimiento del hombro), se han repetido sin y con respaldo de la silla.

Por último, antes de proceder a la ejecución de los ensayos de evaluación con personas, de acuerdo con la normativa de ética del Real Decreto $1716 / 2011$, se ha generado un protocolo de actuación. En este protocolo se establece que en la ejecución de los ensayos de evaluación estarán presentes un técnico que supervise el dispositivo robótico y un fisioterapeuta que se ocupe de comprobar que el participante realice los ejercicios de forma adecuada y segura. De esta forma, se garantiza la correcta ejecución de los ensayos de evaluación.

Antes de comenzar los ensayos de evaluación, a los participantes se les ha proporcionado un documento que describe las pruebas que se llevarán a cabo. Asimismo, se les ha pedido que firmen una hoja de consentimiento en el que han otorgado permiso por escrito para el uso de los datos obtenidos con fines científicos. Finalmente, con el objetivo de detectar aspectos de mejora, al finalizar los ensayos de evaluación, se les ha realizado un breve cuestionario de satisfacción del uso del dispositivo robótico.

\section{ANÁLISIS Y DISCUSIÓN DE LOS RESULTADOS}

Una vez ejecutados los ensayos de evaluación, a continuación se analizan los resultados obtenidos a través del software desarrollado para el análisis de la postura del tronco. En este análisis se han tenido en cuenta los dos objetivos planteados por los fisioterapeutas de este tipo de terapias: 1) Análisis de la postura del tronco de los participantes; 2) Análisis de la contribución del tronco.

\subsection{Análisis de la postura del tronco}

Con la intención de analizar la postura del tronco de los participantes durante la ejecución de los ensayos de evaluación, se analiza la inclinación del tronco de los participantes en la orientación mediolateral (eje $z$ ) y en la fronto-trasera (eje $x$ ). En la Figura 6 se puede observar la inclinación del cuerpo del Participante $A$ con y sin restricciones y con el respaldo de la silla. En azul se observa el rango de inclinación sin restricciones; en rojo con la restricción del codo y en verde con la restricción del hombro.

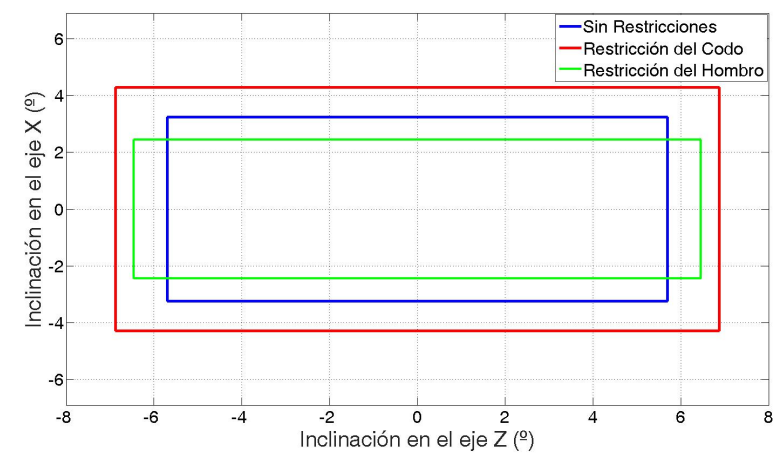

Figura 6: Inclinación del Participante $A$ con respaldo y con y sin restricciones físicas.

Como se puede observar en la Figura 6 y en el resto de ensayos ejecutados con los 25 participantes, los usuarios no han sido capaces de mantener su postura del tronco constante. Por ejemplo, sin restricciones, el Participante $A$ se ha inclinado \pm 5 grados en el eje $z$ (mediorateral) y \pm 3 grados en el eje $x$ (fronto-trasera), mientras que con la restricción del codo la inclinación máxima ha sido de \pm 7 grados.

Además, hay que considerar que estas pruebas se han llevado a cabo con personas sanas, que poseen más capacidad de control del movimiento que los pacientes con movilidad reducida debido a un ataque de ictus. Por lo que se confirma la necesidad de monitorizar la postura adoptada por los pacientes durante las terapias de rehabilitación de miembros superiores mediante dispositivos robóticos.

\subsection{Análisis de la contribución del tronco}

Con el fin de evaluar la necesidad de estimar la contribución del tronco en terapias de rehabilitación, se ha estimado el porcentaje de movimiento ejecutado con el tronco por los 25 participantes con y sin restricciones físicas (Figura 5). Pero antes de realizar este análisis, con la intención de observar que las restricciones diseñadas limitan adecuadamente el rango de movimiento de la extremidad superior de los participantes e identificar las regiones en los cuales su repercusión es mayor, se observan los movimientos generados por los participantes con las restricciones del codo y del hombro. En la Figura 7 se muestran los movimientos realizados por el Participante $A$. En azul de muestra el alcance del participante sin restricciones, mientras que el verde es el movimiento realizado por el usuario durante el ensayo de evaluación.

Como se puede observar en la Figura 7 y en el resto de ensayos ejecutados con los 25 participantes, el efecto de las restricciones físicas ha sido mayor en las regiones 1 y 2 del juego de rehabilitación 


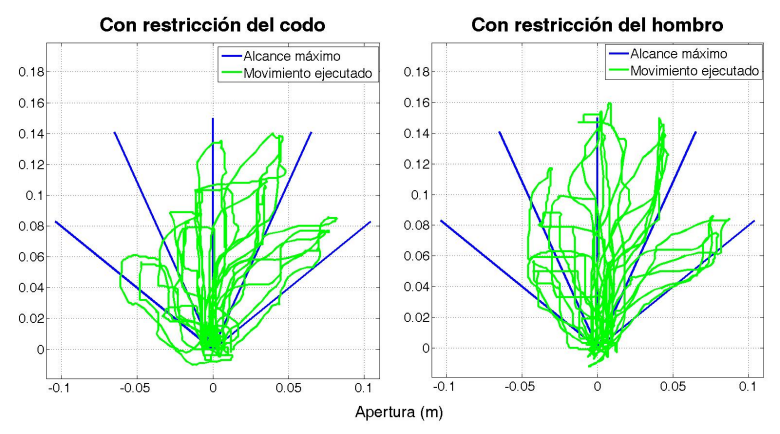

Figura 7: Movimientos ejecutados por el Participante $A$ con las restricciones del codo y del hombro.

(Figura 4). En estas regiones, debido a las restricciones de movimiento, el Participante $A$ no ha sido capaz de llegar ni a la mitad de su amplitud real, mientras que en el resto de regiones ha sido capaz de alcanzar el punto más alejado. Por ello, con el fin de analizar el aporte del tronco durante la ejecución de los ensayos, se ha estimado el porcentaje del movimiento realizado con el tronco del cuerpo en esas dos regiones.

En la Tabla 1 se muestra la media del porcentaje del movimiento ejecutado con el tronco en las regiones 1 y 2 del juego de rehabilitación (Figura 4).

Cuadro 1: Porcentaje del movimiento realizado con el tronco del cuerpo.

\begin{tabular}{cc|c|c}
\hline \multicolumn{2}{c|}{ Ensayo } & Region 1 & Region 2 \\
Restricción & Respaldo & & \\
\hline No & $\mathrm{Si}$ & $3 \%$ & $8 \%$ \\
No & No & $14 \%$ & $7 \%$ \\
Codo & $\mathrm{Si}$ & $27 \%$ & $15 \%$ \\
Codo & No & $36 \%$ & $18 \%$ \\
Hombro & $\mathrm{Si}$ & $30 \%$ & $7 \%$ \\
Hombro & No & $38 \%$ & $13 \%$ \\
\hline
\end{tabular}

Como se detalla en la Tabla 1 , sin restricciones del movimiento, los valores obtenidos han sido menores del $15 \%$, lo que significa que la mayor parte del movimiento se ha ejecutado con la extremidad superior. No obstante, al colocar las restricciones, el porcentaje aumenta notablemente, indicando que los usuarios han utilizado el movimiento del tronco para ejecutar la trayectoria. Lo que confirma la hipótesis inicial la cual afirma que los pacientes con movilidad reducida tienden a inclinar el cuerpo para ayudar al movimiento del miembro superior.

Por otro lado, se observa que el aporte del cuerpo se ha disminuido al introducir el respaldo de la silla. Sin embargo, los participantes han seguido utilizando el tronco para compensar la limitación del movimiento de la extremidad. Por lo tanto, se concluye que un asiento con un respaldo adecuado ayuda a mejorar la posición del tronco de los usuarios, pero no garantiza una posición del tronco adecuada durante la rehabilitación.

Por lo tanto, se verifica la necesidad de conocer el aporte del tronco para asegurar que los pacientes realizan los ejercicios de rehabilitación de forma adecuada y segura.

\section{CONCLUSIONES}

En este trabajo, se ha desarrollado un nuevo software de rehabilitación con capacidad de medir la inclinación del tronco y de la extremidad superior del paciente durante la terapia de rehabilitación mediante un dispositivo robótico. El objetivo del software desarrollado es proporcionar una visualización intuitiva del movimiento del tronco del usuario. Esto le permite al paciente minimizar el error realizado, mientras que el terapeuta puede analizar su comportamiento. Adicionalmente, el software desarrollado estima la contribución del tronco durante la terapia de entrenamiento. Esta estimación brinda al terapeuta una información objetiva que le permite realizar un diagnóstico preciso, ajustar en consecuencia la terapia y monitorizar la evolución en base a datos cuantitativos.

El enfoque desarrollado se ha implementado en el robot de rehabilitación UHP y se ha evaluado realizando diferentes ensayos con 25 personas sanas. Los resultados indican que monitorizar el movimiento del tronco es importante para una rehabilitación exitosa, al mismo tiempo que muestra que el software propuesto funciona correctamente al medir el movimiento del tronco.

Una vez evaluado el software desarrollado con personas sanas que han simulado a usuarios con restricciones de movimiento, en trabajos futuros se pretende evaluar el rendimiento del software con pacientes con déficit motor debido a un ataque de íctus.

\section{Agradecimientos}

Este trabajo ha sido parcialmente financiado por el Ministerio de Economía y Competitividad MINECO \& FEDER en el marco del proyecto DPI2017-82694-R, así como por el proyecto IT914-16 del Gobierno Vasco, el proyecto PPG17/56 de la UPV/EHU y por Euskampus Fundazioa.

Además, los autores desean expresar su agradecimiento al centro de investigación Tecnalia por su colaboración y por prestar su robot de rehabilitación Universal Haptic Pantograph. 


\section{English summary}

\section{ANALYSIS OF THE TRUNK POSTURE IN ROBOT MEDIATED REHABILITATION}

\begin{abstract}
The knowledge of the trunk posture of patients with reduced mobility during rehabilitation therapies, allows better analysis of their recovery state and decrease the recovery times. However, most of upper limb rehabilitation robots are not capable to measure the posture of the trunk. In view of this situation, this work presents an innovate rehabilitation software that allows to therapist to know trunk and affected limb postures of the patient and estimate the percentage of movement performed with the trunk. The proposed software has been implemented in the UHP upper limb rehabilitation robot. Different experimental tests have been carried out with a group of 25 healthy people to demonstrate the validity of the approach. The results show that the proposed software performs correctly when measuring trunk movement.
\end{abstract}

Keywords: Robot mediated rehabilitation, upper limb rehabilitation, trunk posture analysis, experimental validation.

\section{Referencias}

[1] E. Akdo, M. Emin, A. Taha, M. S. Arslan, and M. Atl. Hybrid impedance control of a robot manipulator for wrist and forearm rehabilitation : Performance analysis and clinical results. Mechatronics, 49(July 2017):77-91, 2018.

[2] M. Babaiasl, S. H. Mahdioun, P. Jaryani, and M. Yazdani. A review of technological and clinical aspects of robot-aided rehabilitation of upper-extremity after stroke. Disability and Rehabilitation: Assistive Technology, pages 118, 2015.

[3] J. Bai, A. Song, B. Xu, J. Nie, and H. Li. A Novel Human-Robot Cooperative Method for Upper Extremity Rehabilitation. International Journal of Social Robotics, pages 1-11, 2017.

[4] Z. Corbyn. Statistics: A growing global burden. Nature, 510(7506):S2-S3, 2014.

[5] V. L. Feigin, M. H. Forouzanfar, R. Krishnamurthi, G. a. Mensah, M. Connor, D. A. Bennett, A. E. Moran, R. L. Sacco, L. Anderson, T. Truelsen, M. O'Donnell, N. Venketasubramanian, S. Barker-Collo, C. M. M. Lawes, W. Wang, Y. Shinohara, E. Witt, M. Ezzati, and M. Naghavi. Global and regional burden of stroke during 1990-2010: Findings from the Global Burden of Disease Study 2010. The Lancet, 383(9913):245-255, 2014.

[6] H. M. Hondori and M. Khademi. A Review on Technical and Clinical Impact of Microsoft Kinect on Physical Therapy and Rehabilitation. Journal of Medical Engineering, 2014.

[7] C.-L. Lo and H.-T. Tseng. Predicting rehabilitation treatment helpfulness to stroke patients: A supervised learning approach. Artificial Intelligence Research, 6(2):1, 2017.

[8] A. Mancisidor, A. Zubizarreta, I. Cabanes, P. Bengoa, and J. H. Jung. Kinematical and dynamical modeling of a multipurpose upper limbs rehabilitation robot. Robotics and Computer-Integrated Manufacturing, 49(August 2017):374-387, 2018.

[9] A. Mancisidor, A. Zubizarreta, I. Cabanes, P. Bengoa, and J. H. Jung. Multifunctional robotic device for the rehabilitation of the upper limbs. Revista Iberoamericana de Automática e Informática Industrial, (15):180 191, 2018.

[10] J. L. Pons, R. Raya, and J. González. Emerging Therapies in Neurorehabilitation II. Biosystems \& Biorobotics, 10:29-64, 2016.

[11] T. Proietti, V. Crocher, A. Roby-Brami, and N. Jarrasse. Upper-limb robotic exoskeletons for neurorehabilitation: a review on control strategies. Biomedical Engineering, pages 1$12,2016$.

[12] B. Sheng, Y. Zhang, W. Meng, C. Deng, and S. Xie. Bilateral robots for upper-limb stroke rehabilitation: State of the art and future prospects. Medical Engineering \& Physics, 38(7):587-606, 2016.

[13] A. G. Thrift, D. A. Cadilhac, T. Thayabaranathan, G. Howard, V. J. Howard, P. M. Rothwell, and G. A. Donnan. Global stroke statistics. International Journal of Stroke, 9:6-18, 2014. 
[14] M. Van Eeden, C. M. Van Heugten, and S. M. Evers. The economic impact of stroke in The Netherlands. BMC Public Health, 12(1):122$134,2012$.

[15] Q. Wang, P. Markopoulos, B. Yu, and W. Chen. Interactive wearable systems for upper body rehabilitation : a systematic review. Journal of neuroengineering and rehabilitation, 14(20):1-21, 2017.

[16] S. Xie. Advanced Robotics for Medical Rehabilitation. Springer Tracts in Advanced Robotics, 108:1-357, 2016.

[17] S. Zhang, S. Guo, B. Gao, H. Hirata, and H. Ishihara. Design of a Novel Telerehabilitation System with a Force-Sensing Mechanism. Sensors, 15(5):11511-11527, may 2015.

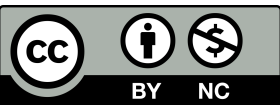

(C) 2018 by the authors. Submitted for possible open access publication under the terms and conditions of the Creative Commons Attribution CC-BY-NC 3.0 license (http://creativecommons.org/licenses/by-nc/3.0/). 